\title{
THE STRUCTURE OF W URSAE MAJORIS SYSTEMS
}

\author{
LeON B. LUCY \\ (Dept. of Astronomy, Columbia University, New York, U.S.A.)
}

According to an argument of Kuiper's, contact binaries with unequal components cannot exist at age zero. This result comes from a comparison of the mass-radius relation for single, homogeneous stars with the condition to be fulfilled by the radii if the surface of the binary is to be an equipotential. This conclusion is contradicted, however, by observational evidence that W UMa stars do occur at age zero with unequal components.

The spectral types of W UMa stars are later than F0, so that their outer layers will be convective. The adiabatic constants of the envelopes of the components must then be equal because the two stars are in contact. Then, since the radius of a star with a convective envelope is sensitive to the adiabatic constant, it follows that the mean radii of the components differ, in this case, from the radii of the corresponding single stars. Thus Kuiper's argument does not apply when the common envelope is convective.

It can then be shown that zero-age contact configurations are possible when the common envelope is convective provided that the $\mathrm{C}-\mathrm{N}$ cycle is dominant in one component and the proton-proton reaction is dominant in the companion. This leads to a model for a W UMa which accounts for some of the properties of these stars.

A paper describing this investigation in detail has been submitted to Astrophys. $J$.

\section{DISCUSSION}

Paczynski: I would like to make two remarks on the problem of the W UMa-type binaries. First, many such binaries show very rapid period variations with $P /(\mathrm{d} P / \mathrm{d} t)$ being of the order of KelvinHelmholtz time-scale. In some systems the orbital period increases, in others it decreases. But in any given binary the sign of the period changes seems to be constant during the last 60 years. I wonder whether these variations are secular or not. Second I have computed the time-scale for the orbital angular-momentum loss due to the emission of gravitational waves from many W UMa-type binaries (cf. Paczyński, Acta Astr., 17, 1967, 00). This time-scale turned out to be very close to the time-scale of nuclear evolution. Perhaps in future, when we shall have a good theory of evolution of the $\mathrm{W}$ UMa-type binaries we shall have a chance to check the physical reality of the gravitational radiation.

Lucy: It would be of interest if an observer would tell us what percentage of W UMa have secular changes in orbital period.

Perek (ed.), Highlights of Astronomy, 452. ㅇ. I.A.U. 